\title{
THE MODULAR FRAMEWORK OF COMPUTER-AIDED VERIFICATION
}

\author{
Gil Shurek and Orna Grumberg \\ Computer Science Department, The Technion \\ Haifa 32000, Israel \\ E-address: orna@techsel (BITNET), orna@sel.technion.ac.il (CSNET)
}

\section{INTRODUCTION}

Temporal logic model checking procedures have been proven to be a feasible approach to automatic verification of relatively small finite-state systems. As the expressive power of temporal logic makes it adequate for specifying properties of reactive systems and as interesting examples are typically those of concurrent and distributed systems, it seems natural to consider the applicability area of automatic model checking as that of reactive finite-state concurrent systems. This context, however, exposes the major disadvantage of model checkers, which is their global nature. By this we mean that only a complete system can be verified to meet its temporal specifications. This globality, on the one hand, induces the "state explosion problem" and, on the other hand, prevents the realization of the "pre-verified component methodology", presented below.

The state explosion problem arises in systems composed of many loosely coupled components. The size of such a system may grow as the product of the sizes of its components. Thus, space requirement might become too large for a model checking procedure to be applicable. Obviously, state explosion affects the time-complexity as well.

The pre-verified component methodology refers to the verification of systems containing an already verified component, that avoids redoing the component's proof. When verifying a specific system, it should be possible to exploit the pre-verification of the component. As a result, the average verification time-complexity over the set of systems containing this preverified component should be reduced.

It seems natural to consider the use of compositional reasoning as a solution to the state explosion problem. Exploiting the modular structure of a system, a compositional verification method applies model checking procedures to individual components, to check properties from which the specification of the global system is deduced. The construction of the global system is avoided, and thus space requirements are reduced. Hopefully time-complexity is reduced as well. The desirability of using pre-verified components raises the need for our method to be modular too. By this we mean that it should be possible to apply a verification procedure to an individual component when no specific environment is available. The difference between compositionality and modularity lies in the amount of information that the verification procedure has about one component when verifying the other. In a compositional approach complete knowledge about the environment may be used, while in the modular approach no information about the actual environment is available. Note that our terminology adapts the terms 
"compositionality" and "modularity", yielding notions that differ slightly from the traditional ones.

This paper presents a modular, semi-automatic method for the verification of finite-state programs with temporal logic specifications. The purpose of this work, however, goes beyond the search for a technical solution to modular verification. The additional goal is to gain a wider understanding of the modular framework. In a related work [SG90] we discuss motivation, solutions, solutions' properties, and evaluation criteria in that context, e.g.:

- An obvious quality of a global model checker that should be preserved in a modular procedure is the ability to verify arbitrary properties of a distributed system, not being restricted to properties of single components within the compound system.

- Modular procedures have no knowledge about the environment. Hence, applying the checking procedure to one component, an assumption on the behavior of the environment is often necessary in order to deduce a global property. Assumptions and their effect on the verification process are thoroughly discussed in [SG90].

Following the previous discussion, this work is a search for a partially automated modular verification procedure supported by a model checking procedure, for reactive, finite-state distributed systems. The systems are modeled by finite structures described as state-transition graphs, where asynchronous actions have an interleaving semantics. We assume that specifications are given by propositional temporal logic formulas that are interpreted over the computation tree of the system's transition graph.

Several works on compositional techniques for reasoning about reactive concurrent systems established the background for our research: [P85],[J87], and [CLM89]. As we regard the "Assume-Guarantee" paradigm [P85] to be the preferable modular scheme, we begin with an exploration of its underlying mechanism. This is done by presenting a deductive proof system scheme $(A G)$ which is an abstraction of that paradigm. Considering a further abstraction (the $A G S$ ) we claim that, such paradigms essentially manipulate infinite sets of models. The main difficulty in implementing an $A G$-like scheme as a semi-automatic procedure, is identified to be the need to represent these sets so that they can be handled automatically.

We present an $A G$-like proof system scheme, denoted $A G M$, in which a set of models is represented by a single model. In this scheme assumptions are expressed by finite statetransition structures, while the specifications are given in a temporal language. The scheme defines complete proof systems, in which soundness depends on conditions that should be satisfied in an actual framework (i.e., an actual model of computation and a temporal language). Implementations of this scheme as semi-automatic procedures are based on an automatic model checker that is not necessarily modular.

We show how a proof system can be derived from the $A G M$ scheme. As the model of computation we choose a simplified version of CCS [M80] which describes asynchronous communicating systems. The specification language is a sublanguage of $C T L^{*}\left(C T L_{-E}^{*}\right)$ which is more expressive than $L T L$. In $C T L_{-E}^{*}$ negation is restricted to the atomic propositions and the $\mathbf{E}$ ("there exists") path quantifier is eliminated. A generalized version of $A G M\left(A G M^{*}\right)$ can be realized in this framework. The $A G M^{*}$ enables simultaneous use of partial information on both components of the distributed system. In $A G M^{*}$, verification can be viewed as state-reduction of the global model without construction of the model. The reduction is relative to the property to be verified (as opposed to minimization which guarantees semantic equivalence of models). Proof systems derived from the $A G M$ and the $A G M^{*}$ schemes can be implemented as semiautomatic procedures for modular verification. Properly used, these procedures enable the use 
of pre-verified components, and guarantee reduced memory requirement and even improved time complexity, compared to a global model checker.

In Section 2 we describe the $A G$ and $A G S$ proof system schemes. In Section 3 the $A G M$ and its generalized version $A G M^{*}$ are presented. Section 4 presents the actual proof system derived from these schemes. In [SG90] this section includes an application of that proof system to a program.

\section{THE AG AND AGS SCHEMES}

\subsection{The $A G$ Proof System Scheme}

The $A G$ deductive proof system scheme presented in this section should be viewed as an abstraction of Pnueli's assume-guarantee paradigm [P85].

Let $\mathbf{L}$ be a logic used as a reasoning and specification language for distributed systems. Let $M$ be a set of models, containing component models and composed models, for which ' $\|$ ' denotes the composition operation. Each composed model $m$ is accompanied with a fixed decomposition into two component models $m_{A}, m_{B}$, such that $m=m_{A} \| m_{B}$. For a component model $m \in M, C_{A}[m]$ denotes the set of all models in $M$ of the form $m_{A} \| m_{B}$, for which $m_{A}$ is $m$ (i.e., $\left.C_{A}[m]=\left(m_{A} \| m_{B} \mid m_{A}=m\right]\right) . C_{B}[m]$ is defined similarly.

For $\varphi_{1}, \varphi_{2} \in L$, the notation $\varphi_{1}{ }_{C_{i}[m]} \varphi_{2}, i \in\{A, B\}$ means that every model in $C_{i}[m]$ which satisfies $\varphi_{1}$, satisfies $\varphi_{2}$ as well. Thus, $\varphi_{2}$ is the consequence of $\varphi_{1}$ in $C_{i}[m], \frac{1}{C_{i}[m]}$ is a proof system that validates $\mathbf{L}$ formulas in $C_{i}[m]$. The soundness of this system is defined as: $\forall \varphi_{1}, \varphi_{2} \in \mathrm{L}\left[\varphi_{1} \frac{1}{c_{i}[m]} \varphi_{2} \Rightarrow \varphi_{1} \varlimsup_{C_{i}[m]} \varphi_{2}\right]$. When $\varphi_{1} \equiv$ true, the definition results in ${ }_{c_{i}(m)} \varphi_{2} \Rightarrow{ }_{c_{i}(m)} \varphi_{2}$ which means that if $\varphi_{2}$ is provable in $\vdash_{c_{i}[m]}$ then $\varphi_{2}$ is true in the composition of $m$ with any environment.

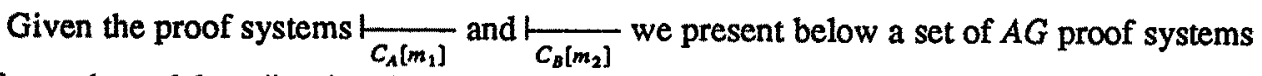
(one for each model $m_{1} \| m_{2}$ ). The proof system $\underbrace{}_{A G-m_{1} \| m_{2}}$ for the verification of L-formulas in the composed model $m_{1} \| m_{2}$ is defined as follows:

Axioms:

$$
\left\{\varphi \mid \underset{c_{A}\left[m_{1}\right]}{\digamma} \varphi\right\} \cup\left\{\varphi \mid \frac{\vdash}{c_{B}\left[m_{2}\right]} \varphi\right\}
$$

Inference rules:

$$
\begin{aligned}
& \vdash_{A G-m_{1} \| m_{2}} \varphi \quad \vdash_{A G-m_{1} \| m_{2}} \varphi \\
& \varphi \vdash_{C_{A}\left[m_{1}\right]} \Psi \quad \varphi \longmapsto_{C_{B}\left[m_{2}\right]} \psi
\end{aligned}
$$

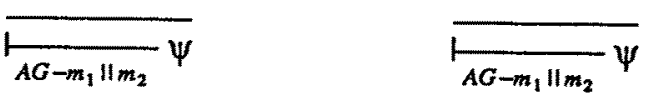

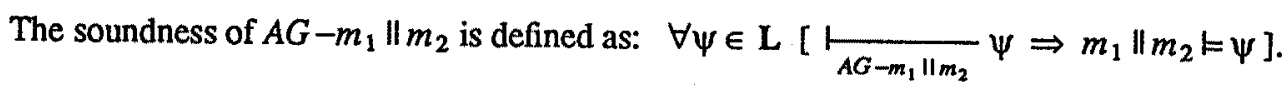


Theorem 1: Given sound proof systems $\frac{\digamma_{A}\left[m_{1}\right]}{}$ and $\longmapsto_{C_{B}\left[m_{2}\right]}, \frac{\vdash_{A G-m_{1} \| m_{2}}}{}$ is sound.

The $A G-m_{1} \| m_{2}$ is a scheme for a proof system, capable of verifying properties of the distributed system $m_{1} \| m_{2}$, without being restricted to the behavior of a single component in the context of the global system. Moreover, it can be considered modular according to our terminology. Examining a typical AG proof, its modularity can be observed:

$$
\vdash_{C_{A}\left[m_{1}\right]} \varphi_{n}, \varphi_{n} \frac{\digamma_{C_{B}\left[m_{2}\right]}}{\varphi_{n-1}} \cdots \varphi_{1} \frac{1}{C_{A}\left[m_{1}\right]} \varphi
$$

We are interested in a semi-automatic implementation of the $A G$ scheme, where the user is required to supply ("guess") the sequence of assumptions $\varphi_{n}$ to $\varphi_{1}$ and the validation in $C_{A}\left[m_{1}\right]$ and in $C_{B}\left[m_{2}\right]$ is done automatically. The $m_{1}$ proof-block is the set of proof stages in which $\frac{}{C_{A}\left[m_{1}\right]}$ is applied. When assumptions are given, the $m_{1}$ proof block can be carried out without having to consider any specific " $m_{2}$ ". Given a component model $m_{i}$, if the proofblock consisting of all $\vdash_{C_{B}[]}$ stages can be carried out successfully with respect to $\frac{1}{C_{B}\left[m_{i}\right]}$, then $m_{1}$ proof-block can be completed to a full $A G$ proof, deducing $m_{1} \| m_{i} \vDash \varphi$.

\subsection{The $A G$-sets ( $A G S$ ) Proof System Scheme}

In this section we present another proof system scheme, $A G$-sets (AGS), which is an abstraction of $A G$, meant to explore the underlying mechanisms of $A G$. In $A G S$, sets of models are used directly as the reasoning language (similar to the Semantic Model of [AL89]). However, we still assume temporal specification. Therefore, an inference rule that derives $\mathbf{L}$ formulas (from a previously "derived" model set) is necessary. Defined below is an AGS proof system for $m_{1} \| m_{2}$.

$\mathrm{gs}\left[\mathbf{m}_{1} \| \mathbf{m}_{2}\right]$ is a set of model sets, defined inductively by:

Atoms ("axioms"):

$$
\left\{C \mid C \supseteq C_{A}\left[m_{1}\right]\right\} \cup\left\{C \mid C \supseteq C_{B}\left[m_{2}\right]\right]
$$

Closure operations ("inference rules"):

$$
\begin{array}{ll}
C \in g s\left[m_{1} \| m_{2}\right] & C \in g s\left[m_{1} \| m_{2}\right] \\
C^{\prime} \supseteq C \cap C_{A}\left[m_{1}\right] & C^{\prime} \supseteq C \cap C_{B}\left[m_{2}\right] \\
\hline C^{\prime} \in g s\left[m_{1} \| m_{2}\right] & \frac{C^{\prime} \in g s\left[m_{1} \| m_{2}\right]}{}
\end{array}
$$

The inference rule:

$$
\begin{aligned}
& \varphi \in \mathbf{L} \\
& C \in g s\left[m_{1} \| m_{2}\right] \\
& =\varphi \\
& \varlimsup_{A G S-m_{1} \| m_{2}} \varphi
\end{aligned}
$$


Theorem 2: $A G S-m_{1} \| m_{2}$ is sound and complete.

Noticing that both $A G$ and $A G S$ basically manipulate potentially infinite sets of models, leads us to identify the major obstacle to the implementation of $A G$. In $A G$, sets of models are represented either by themselves $\left(C_{A}\left[m_{1}\right]\right.$ and $\left.C_{B}\left[m_{2}\right]\right)$ or by L-formulas (i.e., $\varphi$ defines and represents the set of all models satisfying $\varphi$ ). The lack of uniform representation for these sets forces $A G$ implementations (e.g., [J87]) to provide conversion procedures (which are, in general, cumbersome and time-consuming) to enable constructive manipulation of model sets. In $A G S$ we abstract away from the representation problem. The $A G S$ proof system is theoretic and has no explicit representation for sets. This system is not intended to be implemented directly, but to inspire the definition of other proof system schemes.

In the next section we present an AGS-like proof system, in which sets are represented in a way they can be handled constructively.

\section{THE $A G$-MODELS ( $A G M$ ) SCHEMES}

\subsection{The AGM Proof System Scheme}

The $A G M$ is an $A G S$-like proof system scheme, in which the basic elements handled by the deductive procedure are models (i.e., finite state-transition structures). These basic elements are used to represent the potentially infinite sets of models, discussed in the previous section. The $A G M$ proof system is a modular model checking system for finite state distributed system, based on their decomposition into two components.

Let $M$ be a model domain consisting of component models $m_{i}$ and composed models $m$, which may differ syntactically. Let $\Sigma$ be an alphabet and let $\Sigma_{m}$ denote the alphabet associated with the model $m$. $\Sigma_{m}$ consists of state labels and/or transition labels. Let II denote a composition operator that applied to two component models $m_{i}, m_{j}$ results in a composed model $m$, i.e., $m=m_{i} \| m_{j}$. Also, let $\left.\right|_{\Sigma}$ denote a restriction transformer, that applied to a composed model results in a component model with alphabet set restricted to $\Sigma$.

Let $\mathbf{L}$ be a temporal language interpreted over the domain of models $M$. Let $\mathbf{L}(\Sigma)$ denote the set of formulas in $L$ defined over the set of atomic propositions in $\Sigma$. We assume (for the semantics of $L$ ) that if $\varphi \notin L\left(\Sigma_{m}\right)$ then $m \notin \varphi$.

Let $\sqsupseteq$ denote a Preorder (reflexive and transitive relation) defined over $M$, which has the following semantic property: $m_{A} \sqsupseteq m_{B}$ implies that all $\mathrm{L}$ formulas that are satisfied in $m_{A}$, are satisfied in $m_{B}$ (i.e., if $m_{A} \sqsupseteq m_{B}$ than $\forall \varphi \in \mathbf{L}, m_{A} \vDash \varphi \Rightarrow m_{B} \vDash \varphi$ ).

Let $\longmapsto_{M C}$ denote a proof system for checking the satisfaction of $L$ formulas in $M$ models (i.e., a model checker).

The $A G M$ definition inherits the structure of the $A G S$ definition, where inference rules which do not deduce temporal formulas, are referred to as closure operations. Defined below is an $A G M$ proof system for $m_{1} \| m_{2}$.

$\mathbf{g m}\left[\mathbf{m}_{1} \| \mathbf{m}_{2}\right]$ is a set of models, defined inductively by:

Atoms : $\quad\left(m^{\prime} \| m_{2} \mid m^{\prime} \sqsupseteq m_{1}\right) \cup\left\{m_{1} \| m^{\prime \prime} \mid m^{\prime \prime} \sqsupseteq m_{2}\right\}$

closure operations : 


$$
\begin{array}{lc}
m_{1} \| m^{\prime \prime} \in g m\left[m_{1} \| m_{2}\right] & m^{\prime} \| m_{2} \in g m\left[m_{1} \| m_{2}\right] \\
\left.m^{\prime} \sqsupseteq\left(m_{1} \| m^{\prime \prime}\right)\right|_{\Sigma_{m_{1}}} & \left.m^{\prime \prime} \sqsupseteq\left(m^{\prime} \| m_{2}\right)\right|_{\Sigma_{m_{2}}} \\
\hline m^{\prime} \| m_{2} \in g m\left[m_{1} \| m_{2}\right] &
\end{array}
$$

The inference rule :

$$
\begin{aligned}
& m \in g m\left[m_{1} \| m_{2}\right] \\
& \varphi \in \mathbf{L}\left(\Sigma_{m}\right) \\
& F_{M C} m=\varphi \\
& \frac{F_{A G M-m_{1} \| m_{2}} \varphi}{}
\end{aligned}
$$

The soundness of the $A G M$ scheme depends on the following conditions ( regarding the language, the models domain, the composition operator, the restriction transformer and the semantic preorder) :

\section{Soundness Conditions :}

1. For all $m^{\prime}, m_{A}, m_{B}$ component models in $M$, if $m_{A} \sqsupseteq m_{B}$ then $m_{A}\left\|m^{\prime} \sqsupseteq m_{B}\right\| m^{\prime}$.

2. For all $m^{\prime}, m_{A}, m_{B}$ component models in $M$, if $\left.m_{A} \| m^{\prime}\right] m_{B} \| m^{\prime}$ then

$$
\left.\left.\left(m_{A} \| m^{\prime}\right)\right|_{\Sigma_{m}} \sqsupseteq\left(m_{B} \| m^{\prime}\right)\right|_{\Sigma_{m}} \text {. }
$$

3. For all $m_{A}, m_{B}$ component models in $M, \quad\left(\left.\left(m_{A} \| m_{B}\right)\right|_{\Sigma_{m_{A}}}\right)\left\|m_{B} \supseteq m_{A}\right\| m_{B}$.

When the composition operator is not commutative the dual conditions are required as well.

Theorem 3: Given that the soundness conditions are fulfilled then:

\subsection{Using a sound proof system $\vdash_{M C}, \frac{\vdash_{A G M-m_{1} \| m_{2}}}{}$ is sound.}

3.2 Using a complete proof system $\frac{}{M C}, \frac{1}{A G M-m_{1} \| m_{2}}$ is complete.

Comment: Systems which are composed of more than two components, can be handled by $A G M$-like schemes.

\subsubsection{Evaluation}

The $A G M$ scheme is capable of verifying any property of the global distributed system, which is expressible in L. Examining a typical $A G M-m_{1} \| m_{2}$ proof, verifying that $m_{1} \| m_{2} \vDash \varphi$, its modularity can be observed. Regarding the state explosion problem, this proof system is feasible as long as the size of the structures representing the assumptions is significantly less than the size of the components' models. Applying the pre-verified components methodology, feasibility depends on the assumptions strength. As "greater" ( $]$ ) structures make weaker assumptions, the $A G M$ enables the user to weaken the supplied assumptions provided that the knowledge needed for the verification of the temporal specification is preserved.

\subsubsection{Resemblance to the AGS Scheme}

In the $A G M$ scheme, a single model of a composed system represents an $A G S$ basic element: a set of models. The model represents the set of composed models in $M$ which are 
smaller with respect to the semantic relation $\sqsupseteq \quad$ (i.e., $m$ represents a set of models in which every formula satisfied by $m$ is valid). The "greater" model can be viewed as holding partial information about the "small" one. Thus, the preorder between two basic elements of the AGM is analogous to the subset relation between $A G S$ sets. The task of combining partial knowledge about the checked system with full information about one of the components, which is carried out by the intersection operator in the $A G S$ scheme, is achieved here by applying the composition operator itself (aided by the restriction transformer).

\subsubsection{Implementation}

A computer-aided implementation of the $A G M$ proof system is expected to carry out automatically the following actions: a. Models composition, b. Applying the restriction transformer, c. Verifying the satisfaction of $\mathbf{L}$ formulas in $M$ models, d. Verifying the preorder between models.

It is not difficult to imagine implementable definitions of a composition operator and a restriction transformer which make tasks a. and b. algorithmic. To automate task c. , a global model checker can be applied. The only task which is not clearly decidable even for the finite-state case is the verification of the semantic preorder (task d. ). Any useful implementation of the $A G M$ system should suggest a semantic preorder which is syntactically identified by an efficient algorithm.

In section 4. we suggest an implementation of $A G M$ system in which all four tasks are algorithmically handled. As can be seen there, the need for a decidable preorder affects the choice of the temporal specification language.

\subsection{The $A G M^{*}$ Proof System Scheme}

Setting stricter soundness conditions, the $A G M^{*}$ which is a generalized version of $A G M$, can be defined. The $A G M^{*}$ enables simultaneous use of partial information on both components of the distributed system. Using this scheme it is possible to conduct a proof, along which only the knowledge that is essential for the verification of the final specification is preserved.

$\mathrm{gm}^{*}\left[\mathbf{m}_{1} \| \mathbf{m}_{2}\right]$ :

Atoms : $\quad\left\{m_{A} \| m_{B} \mid m_{A} \sqsupseteq m_{1}, m_{B} \sqsupseteq m_{2}\right\}$

Closure operation:

$$
\begin{aligned}
& \bar{m}, \bar{m} \in g m^{*}\left[m_{1} \| m_{2}\right] \\
& \left.m^{\prime} \sqsupseteq \bar{m}\right|_{\Sigma_{m_{1}}} \\
& \left.m^{\prime \prime} \sqsupseteq \overline{\bar{m}}\right|_{\Sigma_{m_{2}}} \\
& \overline{m^{\prime} \| m^{\prime \prime} \in g m^{\prime \prime}\left[m_{1} \| m_{2}\right]}
\end{aligned}
$$

The inference rule : 


$$
\begin{aligned}
& m \in g m^{*}\left[m_{1} \| m_{2}\right] \\
& \varphi \in \mathbf{L}\left(\Sigma_{m}\right) \\
& \stackrel{M C}{{ }_{M}} m \varphi \\
& \underset{A G M^{*}-m_{1} \| m_{2}}{\longmapsto}
\end{aligned}
$$

In the case where "smaller" ( $\sqsupseteq$ ) models can often be represented by smaller structures, the $A G M^{*}$ enables further reduction in the size of the structures handled by the proof system (in comparison with $A G M$ ). Thus, further reduction in time and space complexity is achieved.

\section{ASYNCHRONOUS COMMUNICATING SYSTEMS}

\subsection{The Model of Computation}

We use here a simple computation model for distributed systems. The components of these systems are asynchronous processes, that communicate through synchronized actions. This model is similar to the CCS model [M80]. Our version is restricted to one application of the composition operation (i.e., every model of a compound system is composed of two process models). In this version, compound models are syntactically guaranteed to be deadlock free, i.e., when a composed system is verified using this type of models, all the deadlocked sub-structures of the computation tree of the system are ignored.

\subsection{The Specification Language}

Defined below is a propositional temporal logic which is a sublanguage of $C T L^{*}$ [CE81]. Let $\Sigma$ be the disjoint union of $A P_{\Sigma}$ and $A C_{\Sigma}$. The language $C T L_{-E}^{*}(\Sigma)$ is defined as the smallest set of state formulas such that:

(1) If $A \in A P_{\Sigma}$, then $A$ and $\neg A$ are state formulas.

(2) If $\varphi$ and $\psi$ are state formulas, then $\varphi \vee \psi$ and $\varphi \wedge \psi$ are state formulas.

(3) If $\varphi$ is a path formula, then $\mathbf{A} \varphi$ is a state formula.

(4) If $\varphi$ is a state formula, then $\varphi$ is a path formula.

(5) If $\varphi$ and $\psi$ are path formulas, then $\varphi \vee \psi, \varphi \wedge \psi, \varphi \mathrm{U} \psi, \mathrm{X} \varphi$ and $\mathrm{G} \varphi$ are path formulas.

Note that in $C T L_{-E}^{*}$ negations are applied only to atomic formulas. Thus, the path quantifier $\mathbf{E}$ ("for some computation path") is eliminated semantically from $C T L_{-E}^{*}$. The semantics of $C T L_{-E}^{*}\left(\Sigma_{m}\right)$ is defined in the usual way, with respect to the Kripke structure $K_{m}$ [HC77], generated from the (process or system) model $m$ by ignoring the model's transition labels.

The satisfaction of a state formula $\varphi \in C T L_{-E}^{*}(\Sigma)$ in a model $m$ (with $s_{0}$ the initial state), denoted $m \vDash \varphi$, is defined by :

(1) If $\varphi \in C T L_{-E}^{*}\left(\Sigma_{m}\right)$ then $m \vDash \varphi$ iff $m, s_{0} \vDash \varphi$.

(2) If $\varphi \notin C T L_{-E}^{*}\left(\Sigma_{m}\right)$ then $m \sharp \varphi$.

We say that one language is more expressive then the other if there exists a formula in the one that does not have an equivalent formula in the other. Let $\zeta$ denote "more expressive" and let $C T L_{-E}$ be $C T L \cap C T L_{-E}^{*}$. Comparing $C T L_{-E}^{*}$ with $C T L^{*}, C T L$ and $L T L$ 
[CG87] we get the following lemma :
Lemma 3: (a) $\left.C T L^{*}\right\} C T L_{-E}^{*}$;
(b) $\left.C T L_{-E}^{*}\right\} C T L_{-E}$;
(c) $\left.C T L_{-E}^{*}\right\}$ LTL.

We can now choose a model checking procedure $\vdash_{M C}$, needed in our modular proof systems ( $A G M$ and $\left.A G M^{*}\right)$. As $C T L_{-E}^{*}$ is a sub language of $C T L^{*}$ there is a model checking algorithm which is exponential in the size of the specification and linear in the size of the Kripke structure (i.e., $\left.O\left(2^{\mid \varphi !}(|S|+|R|)\right)\right)$ [EL85]. One may prefer to restrict the specifications to $C T L_{-E}$, gaining a linear model checking algorithm (i.e., $\left.\mathrm{O}(|\varphi|(|S|+|R|))\right)$ [CES86].

\subsection{The Preorder}

The choice of $C T L_{-E}^{*}$ as a specification language is motivated by a unique semantic quality: The validity of $C T L_{-E}^{*}$ formulas is preserved over "pruned" computation trees. A description of this property is presented below. In this context, we view the semantics of $C T L_{-E}^{*}$ as defined over computation trees. The originating transition graphs (if exist) of these trees are omitted. Let $T$ be a computation tree, and $\varphi \in C T L_{-E}^{*}$ be a temporal formula. $T, v \vDash \varphi$ denotes that $\varphi$ is satisfied at node $v$ in $T$. A branching node in $T$ is a node that has at list two immediate descendants. Eliminating a subtree of $T$ rooted at $v$, such that $v$ is an immediate descendant of a branching node, is defined as pruning $T$ at $v$.

Theorem 5: If $T^{\prime}$ is generated by pruning $T$ at nodes which are descendants of $v$, then $\forall \varphi \in C T L_{-E}^{*}\left[T, v \vDash \varphi \Rightarrow T^{\prime}, v \vDash \varphi\right]$.

Long [L89] defined a syntactic preorder over Kripke structures, which partially identifies the pruning relation of their computation trees. Basically, we adopt his relation as our choice of the required semantic preorder $\sqsupseteq$. Assimilated in our system, the definition is enhanced to encompass more cases for which the semantic property holds, including more cases for which the pruning relation holds. In addition, it is modified to consider transition labels and to verify the containment of the atomic proposition sets.

Let $m=\left\langle A C, A P, S, R, s_{0}, L\right\rangle$ and $m^{\prime}=\left\langle A C^{\prime}, A P^{\prime}, S^{\prime}, R^{\prime}, s_{0}^{\prime}, L^{\prime}\right\rangle$ be either both process models, or both system models, such that $A P \subseteq A P^{\prime}$. A sequence of relations $F_{i}$, such that $\forall i \geq 0 \quad F_{i} \subseteq S \times S^{\prime}$, is defined as follows:

For all $s \in S, s^{\prime} \in S^{\prime}$

1. $s F_{0} s^{\prime}$ iff $L(s)=L^{\prime}\left(s^{\prime}\right) \cap A P$.

2. $s F_{n+1} s^{\prime}$ iff .

$2.1 s F_{n} s^{\prime}$.

$2.2 \forall s_{1}^{\prime} \in S^{\prime}$, for all action $\alpha\left[s^{\prime} \stackrel{\alpha}{\longrightarrow} s_{1}^{\prime} \Rightarrow \exists s_{1} \in S\left[s \stackrel{\alpha}{\longrightarrow} s_{1} \wedge s_{1} \boldsymbol{F}_{n} s_{1}^{\prime}\right]\right]$. $F \subseteq S \times S^{\prime}$ is defined by: For all $s \in S, s^{\prime} \in S^{\prime}, \quad s F s^{\prime}$ iff $\forall i \geq 0 \quad s F_{i} s^{\prime}$. Let $m=\left\langle A C, A P, S, R, s_{0}, L\right\rangle$ and $m^{\prime}=\left\langle A C^{\prime}, A P^{\prime}, S^{\prime}, R^{\prime}, s_{0}^{\prime}, L^{\prime}\right\rangle$. The relation $\geq$ over the model domain $M$ is defined as follows:

$m \geq m^{\prime}$ iff -

i. $\quad m$ and $m^{\prime}$ are either both process models, or both system models.

ii. $A P \subseteq A P^{\prime}$.

iii. $s_{0} F s_{0}^{\prime}$.

Theorem 6: If $m_{1} \geq m_{2}$ then for every $\Sigma$, and for every formula $\varphi \in C T L_{-E}^{*}(\Sigma)$, $m_{1} \vDash \varphi \Rightarrow m_{2} \vDash \varphi$. 
There is an algorithm to verify that two models are preordered, which is polynomialtime in their size. Using $\geq$ as the required syntactically-identified semantic preorder, note that "greater" $(\geq)$ models are, in general, expressed by smaller structures. The implications of this property were previously discussed.

Theorem 7: The $A G M$ (and $A G M^{*}$ ) soundness conditions are satisfied by the model of computation, the language $C T L_{-E}^{*}$, and the preorder $\geq$.

Establishing that, the presentation of a semi automatic model checking system derived from these schemes, is completed. In [SG90] we apply these proof rules to a program.

\section{REFERENCES}

[AL89] M. Abadi and L. Lamport, "Composing Specifications", REX Workshop on Stepwise Refinement of Distributed Systems: Models, Formalisms, Correctness, Mook, May 1989.

[CE81] E.M. Clarke, and E.A. Emerson, "Synthesis of Synchronization Skeletons for Branching Time Temporal Logic", Proc. of Workshop on Logic of Programs, Yorktown-Heights, 1981.

[CES86] E.M. Clarke, E.A. Emerson, and A.P. Sistla, "Automatic Verification of FiniteState Concurrent Systems using Temporal Logic Specifications", ACM Transactions on Programming Languages and Systems 8, 2, pp. 244-263, 1986.

[CG87] E.M. Clarke, O. Grumberg, "Research on automatic verification of finite-state concurrent systems", Annual Reviews of Computer Science, Vol. 2, 269-290, 1987 (J.F. Traub, editor).

[CLM89] E.M. Clarke, D.E. Long, and K.L. McMillan, "Compositional Model Checker". Proc. of the 4th IEEE Symp. on Logic in Computer Science, Asilomar, June 1989.

[EL85] E.A. Emerson, and C. Lei, "Modalities for Model Checking: Branching Time Strikes Back", 12th Symposium on Principles of Programming Languages, New Orleans, La., January 1985.

[HC77] G.E. Hughes, and M.J. Creswell, "An introduction to Modal Logic", London: Methuen, 1977.

[J87] B. Josko, "MCTL - An Extension of CTL for Modular Verification of Concurrent Systems". Workshop on Temporal Logic, (H. Barringer, ed.) University of Manchester, April 1987, LNCS 398, pp. 165-187.

[L89] D.E. Long, Private communication.

[M80] R. Milner, A Calculus of Communicating Systems, Springer Lecture Notes on Computer Science, Vol. 92, 1980.

[P85] A. Pnueli, "In Transition from Global to Modular Temporal Reasoning about Programs". Logics and Models of Concurrent Systems, (K. Apt, ed.), Vol. 13 of NATO ASI Series F: Computer and System Sciences, Springer-Verlag, 123-144,

[SG90] G. Shurek and O. Grumberg, "THE MODULAR FRAMEWORK OF COMPUTER-AIDED VERIFICATION Motivation, Solutions, and Evaluation Criteria", Workshop on Computer-Aided Verification, Rutgers, NJ., June 1990, to appear in ACM/AMS DIMACS series. 